noise exposure levels, hearing levels, depression, anxiety, burnout symptoms, work-related stress and tinnitus will be analysed by use of multiple logistic regression analysis, taking a priori selected potential confounders into account.

Results Preliminary results show that of the 554 participants, $77 \%$ were males an the mean age was 43 years, ranging from 20-64 years. Among the participants, 17\% reported tinnitus, $16 \%$ had a hearing handicap (WHO-definition), 3\% anxiety disorder, 14\% burn-out symptoms, $4 \%$ depression, and $8 \%$ reported work-related stress.

Conclusions Tinnitus is expected to represent a heterogeneous group of underlying disorders. We aim at contributing to a better understanding of the relative importance of the possible underlying factors in a population of occupationally noise exposed workers. Risk analyses are in progress, and results will be presented at the conference.

\section{THE TREND IN THE PREVALENCE OF CHILDHOOD HEARING IMPAIRMENT IN TAIWAN AND ITS IMPLICATIONS}

\begin{abstract}
1,2 2 Der-Chung Lai, ${ }^{3}$ Yen-Cheng Tseng, ${ }^{4,5}$ Cheng-Yu Lin, ${ }^{5,6}$ How-Ran Guo. ${ }^{1}$ Ditmanson Medical Foundation Chia-Yi Christian Hospital, Chia-Yi, Taiwan; ${ }^{2}$ Chia Nan University of Pharmacy and Science, Tainan, Taiwan; ${ }^{3}$ Chang Jung Christian University, Tainan, Taiwan; ${ }^{4}$ Tainan Hospital, Ministry of Health and Welfare, Tainan, Taiwan; ${ }^{5}$ National Cheng Kung University Hospital, Tainan, Taiwan; ${ }^{6}$ National Cheng Kung University, Tainan, Taiwan
\end{abstract}

\subsection{6/oemed-2014-102362.211}

Objectives Childhood hearing impairment (CHI) is a major developmental disability, but data at the national level are limited, especially those on the trends over time. We conducted a study to assess the time trend of CHI prevalence in Taiwan and explore its associated factors.

Method The Taiwan government certifies disabled residents for providing various services and maintains a registry of certified cases. We analysed the registry data on cases under 17 years old from 2000 to 2011 to estimate the prevalence of CHI and assess the time trend.

Results Each year, registered cases ranged from 3427 to 4075 . The prevalence increased from 2000 to 2006, and then decreased till 2011. In general, the prevalence increased over the years in the age groups $<3$ years, 3-5 years, and 6-11 years $(p<0 \cdot 05)$, but decreased in the age group 12-14 years $(p<0.05)$. The largest increase was observed in the age group $<3$ years, particularly after the promotion of screening by the government in 2003. The decrease after 2006 was mainly attributable to decreases in the age groups $12-14$ and $15-17$ years, and similar decreases had been observed in countries with rubella vaccination programs.

Conclusions In Taiwan, the prevalence and proportion of $\mathrm{CHI}$ in the age group $<3$ years had increased from 2000 to 2006 after the implementation of hearing screening programs. The overall prevalence decreased from 2006 to 2011, which might be attributable to the improvements in medical care and the nationwide rubella vaccination program.

\section{A STRUCTURAL EQUATION MODELLING METHOD TO DESCRIBE WORK ENVIRONMENT RISK FACTORS AND MUSCULOSKELETAL SYMPTOMS AMONG HOSPITAL NURSES}

Alireza Dehdashti. Semnan University of Medical Sciences, Semnan, Iran
Objectives Epidemiological studies have shown that musculoskeletal symptoms are frequent occupational injury and disability among nurses in developing countries. Preventive measures were not able to reduce musculoskeletal disorders significantly in nursing profession. This study was performed to provide a comprehensive assessment of the association among risk factors in developing musculoskeletal symptoms.

Method A structural equation model was applied to describe and analyse complex causal relationships from sets of occupational variables involved in musculoskeletal symptoms. A questionnaire was applied to nurses at hospitals affiliated to Semnan Medical Sciences University to collect data on personal and occupational factors and musculoskeletal symptoms. An ergonomic rapid entire body assessment measured work posture risks.

Results Physical work demand and mental pressure increased the risk of musculoskeletal disorders significantly. Musculoskeletal symptoms increased in nurses who worked for extended hours and experienced frequent unstable work posture. Structural equation model showed that musculoskeletal symptoms were associated directly and indirectly by physical and mental job variables, employment status, age and lifestyle.

Conclusions Covarience structural analysis is useful to describe and understand both the direct and indirect effects of variables with complex relationship between risk factors on the prediction of musculoskeletal symptoms.

\section{PTSD AND COGNITIVE IMPAIRMENT AMONG RESCUE AND RECOVERY WORKERS EXPOSED TO THE 9/11 WORLD TRADE CENTRE DISASTER}

${ }^{1}$ Sadie Sanchez, ${ }^{1}$ Stephanie Barnhart, ${ }^{2}$ Steven Stellman, ${ }^{2}$ James Cone, ${ }^{2}$ Robert Brackbill. ${ }^{1}$ Icahn School of Medicine at Mount Sinai, New York, NY, USA; ${ }^{2}$ World Trade Center Health Registry (NYCDOHMH), New York, NY, USA

\subsection{6/oemed-2014-102362.213}

Objectives The 9/11 World Trade Centre Disaster (WTCD) has resulted in many chronic health problems among survivors. Rescue/recovery workers (RRW), having the highest exposures, would likely be at the highest risk for these problems. We sought to evaluate cognitive impairment among RRW with and without prevalent posttraumatic stress disorder (PTSD) which may act as a mediator.

Method The World Trade Centre Health Registry is a voluntary longitudinal cohort study. Surveys collected demographics as well as 9/11 related exposures and health outcomes. We limited analysis to RRW workers only. WTCD exposure among RRW was classified from low to very high based on a sum of twelve 9/ 11 exposures. Cognitive impairment was defined by the frequency of trouble remembering things over the prior 7 days, and presence and/or worsening of memory loss or confusion over the prior 12 month period. A PTSD checklist score of 44 or greater was used to assess the presence of probable PTSD.

Results Of 18932 RRW, 2436 (12.9\%) had PTSD while 16506 (87.1\%) did not have PTSD. Among those without PTSD, 7.9\% in the low exposure group were cognitively impaired, $8.9 \%$ with medium exposure, $10.3 \%$ with high exposure, and $14.4 \%$ with very high exposure. Among those with PTSD, $33.6 \%$ in the low exposure group were cognitively impaired, $33.5 \%$ with medium exposure, $32.5 \%$ with high exposure, and $38.1 \%$ with very high exposure.

Conclusions In WTCD RRW without PTSD, there is a doseresponse for cognitive impairment with increased exposure. 